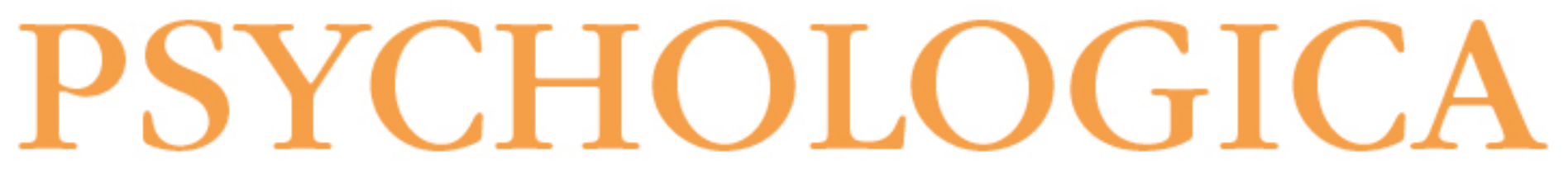

\title{
Relação entre sintomas de insônia em crianças e problemas comportamentais avaliados pelo Child Behavior Checklist (CBCL)
}

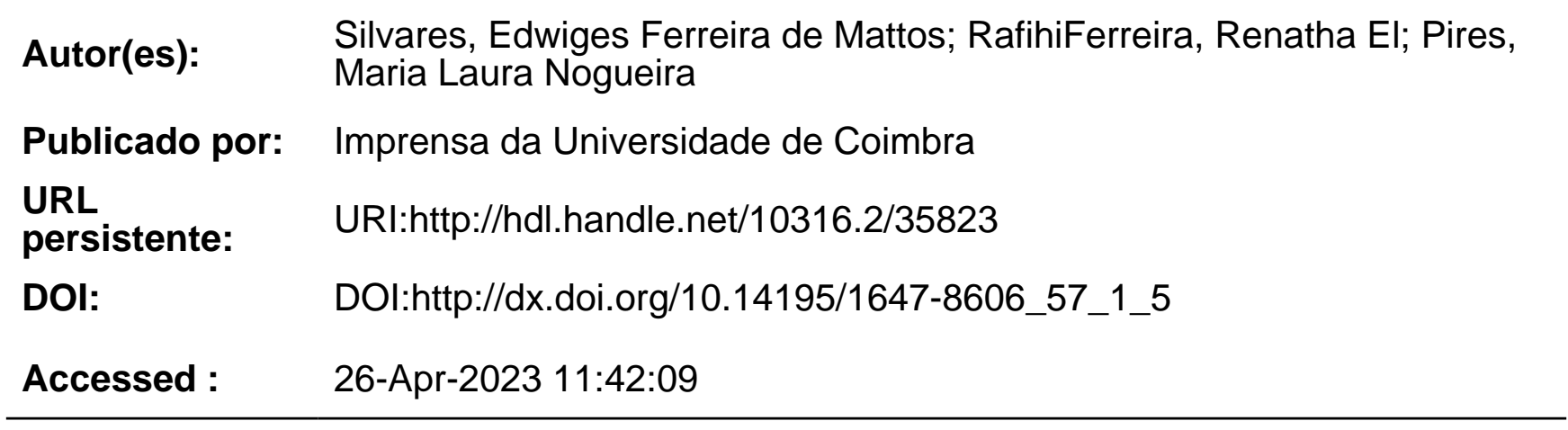

A navegação consulta e descarregamento dos títulos inseridos nas Bibliotecas Digitais UC Digitalis, UC Pombalina e UC Impactum, pressupõem a aceitação plena e sem reservas dos Termos e Condições de Uso destas Bibliotecas Digitais, disponíveis em https://digitalis.uc.pt/pt-pt/termos.

Conforme exposto nos referidos Termos e Condições de Uso, o descarregamento de títulos de acesso restrito requer uma licença válida de autorização devendo o utilizador aceder ao(s) documento(s) a partir de um endereço de IP da instituição detentora da supramencionada licença.

Ao utilizador é apenas permitido o descarregamento para uso pessoal, pelo que o emprego do(s) título(s) descarregado(s) para outro fim, designadamente comercial, carece de autorização do respetivo autor ou editor da obra.

Na medida em que todas as obras da UC Digitalis se encontram protegidas pelo Código do Direito de Autor e Direitos Conexos e demais legislação aplicável, toda a cópia, parcial ou total, deste documento, nos casos em que é legalmente admitida, deverá conter ou fazer-se acompanhar por este aviso.

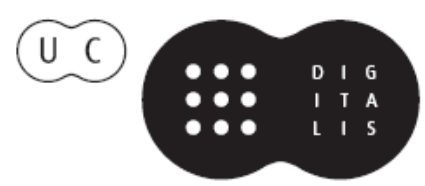


VOLUME $5 \longdiv { 2 0 1 4 }$

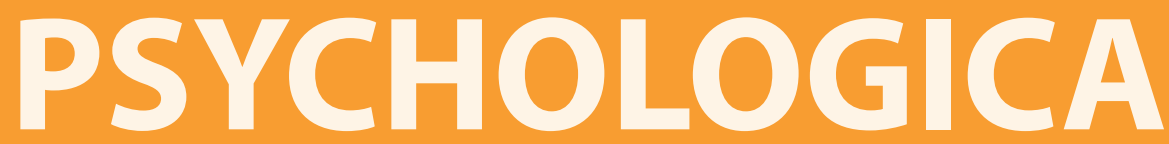

IMPRENSA DA UNIVERSIDADE DE COIMBRA

COIMBRA UNIVERSITY PRESS

FACULDADE DE PSICOLOGIA E DE CIÊNCIAS

DA EDUCAÇÃO DA UNIVERSIDADE DE COIMBRA 


\title{
Relação entre sintomas de insônia em crianças e problemas comportamentais avaliados pelo Child Behavior Checklist ${ }^{1}$ (CBCL)
}

\author{
Edwiges Ferreira de Mattos Silvares ${ }^{2}$, Renatha El Rafihi-Ferreira ${ }^{3}$ e \\ Maria Laura Nogueira Pires ${ }^{4}$
}

\begin{abstract}
Relationship between symptoms of insomnia in children and behavioral problems assessed by the Child Behavior Checklist (CBCL)
\end{abstract}

\begin{abstract}
Sleep problems are common in children and can result in losses in the daytime behavior of a child. This study aimed to present a review of behavioral problems assessed by the instrument Child Behavior Checklist (CBCL) for children with symptoms of insomnia. Studies selected for review in this article were identified through the databases BVS Psicologia ULAPSI Brasil, SCOPUS and PsycINFO, between 2001 and 2011, using the following key words in various combinations: Children, CBCL, Child Behavior Checklist, Behavior, Sleep, Sleep problems, Behavioral Insomnia, Bedtime problems, Night wakings. According to the inclusion criteria of this study, we selected twelve studies. The results of these publications in this review showed that most studies found significant associations between sleep problems and behavior problems, either if they were externalizing, internalizing or other behavioral problems assessed by the CBCL.
\end{abstract}

Keywords: children; trouble sleeping; Child Behavior Checklist; internalizing problems; externalizing problems

1 Inventário de Comportamentos da Infância e Adolescência.

2 Universidade de São Paulo - USP. Email: efdmsilv@usp.br

3 Universidade de São Paulo - USP.

4 Universidade Estadual Paulista - Julio de Mesquita Filho (UNESP campus Assis). 


\section{Resumo}

Problemas de sono são frequentes na população infantil, e podem resultar em prejuízos no comportamento diurno da criança. O presente estudo teve como objetivo apresentar uma revisão sobre problemas comportamentais avaliados pelo instrumento Child Behavior Checklist (CBCL) de crianças com sintomas de insônia. Os estudos selecionados para revisão deste artigo foram identificados por meio das bases de dados BVS Psicologia ULAPSI Brasil, SCOPUS e PsycINFO, entre os anos 2001 e 2011, utilizando as seguintes palavras-chaves em diferentes combinações: Children, CBCL, Child Behavior Checklist, Behavior, Sleep, Sleep problems, Behavioral Insomnia, Bedtime Problems, Night Wakings . De acordo com os critérios de inclusão do presente trabalho, foram selecionados doze estudos. Os resultados das publicações nesta revisão demonstraram que a maior parte das pesquisas encontraram associações significativas entre problemas de sono e problemas de comportamento, fossem eles externalizantes, internalizantes ou outros problemas de comportamento avaliados pelo CBCL.

Palavras-chave: criança; problema de sono; Child Behavior Checklist; problemas internalizantes; problemas externalizantes

\section{INTRODUÇÃO}

A insônia comportamental na criança se manifesta como dificuldade para adormecer quando é colocada na cama ou de permanecer dormindo ao longo da noite, despertando várias vezes e resistindo a voltar a dormir (American Academy of Sleep Medicine, 2005; Owens \& Mindell, 2011).

A insônia comportamental é o distúrbio de sono mais frequente na população pediátrica, atingindo cerca de $25 \%$ a $40 \%$ das crianças (American Academy of Sleep Medicine, 2005; Mindell \& Owens, 2010; Owens \& Mindell, 2011). No estudo brasileiro de Pires, Vilela e Câmara (2012), utilizando uma amostra não clínica, do tipo de conveniência, mães de bebês ( $N=25,1$ ou 2 anos de idade), e de crianças em idade pré-escolar $(N=23$, de 3 a 5 anos de idade) e escolar $(N=$ 46 , idade entre 6 e 12 anos), responderam a versão em português do instrumento The Sleep Disturbance Scale for Children. Os resultados mostraram que 8,0\%, 8,7\% e 13,0\% dos bebês e das crianças em idade pré-escolar e escolar, respectivamente, não obtêm sono com duração recomendada para sua faixa etária. Embora seja esperado que existam variações individuais na necessidade de sono, é particularmente importante o achado de que praticamente uma em cada 10 crianças 
em idade pré-escolar ou escolar dorme menos do que 8 horas por noite. Uma parte importante das mães das crianças em idade pré-escolar $(56,6 \%)$ e escolar $(54,4 \%)$ referiu que seus filhos habitualmente (1 a 2 vezes por semana ou mais) relutam para dormir e aproximadamente um terço das mães afirmou que seus filhos têm dificuldades para dormir e que os percebem sonolentos ao longo do dia. Os problemas rotineiros mais frequentemente citados - resistência para ir para a cama e dificuldades para dormir - superaram, contudo, as estimativas internacionais citadas na literatura especializada (variação de 15\% a 30\%; Mindell \& Owens, 2010).

Contudo, estudos de prevalência da insônia em crianças diferem quanto aos critérios adotados em termos de frequência e gravidade dos sintomas de insônia (por exemplo, número de vezes por semana que a criança desperta, latência para voltar a adormecer, etc). As definições de insônia em crianças tendem a ser baseadas em critérios de inclusão que podem variar desde um relato mais geral ("meu filho tem dificuldade para adormecer") para os mais específicos (leva mais de 20 minutos para adormecer, pelo menos três noites por semana, durante pelo menos três meses). Além disso, os critérios de inclusão podem variar de acordo com a idade e o estado de desenvolvimento da criança (Owens \& Mindell, 2011).

Como o sono é um processo fisiológico e comportamental essencial para a saúde do organismo, os problemas de sono em crianças são fonte de preocupação. O sono é responsável por aproximadamente $40 \%$ do dia típico de uma criança. Quando as crianças não dormem o suficiente, aspectos de seu desenvolvimento físico, emocional, cognitivo, comportamental e social podem ser afetados negativamente (Meltzer \& Mindell, 2008; Witcher et al., 2012). As consequências dos problemas de sono na funcionalidade diurna e nos comportamentos de crianças têm sido foco de várias pesquisas, sendo que alguns estudiosos, como por exemplo, Gregory e Sadeh (2012) e Hill (2011), apontam uma possível associação entre problemas de sono e de comportamento na população infantil.

Para investigar possíveis associações entre sono e comportamento, bem como para o planejamento de práticas preventivas e interventivas, é essencial realizar uma avaliação cautelosa do sono e dos problemas de comportamento da criança.

Para diagnosticar a insônia comportamental pediátrica é necessária uma avaliação clínica. Nesta avaliação, devem ser identificadas informações que contemplem vários pontos como: a) os horários de dormir e acordar; b) o tempo que a criança leva para adormecer; c) o número e o tempo dos despertares noturnos; d) a duração do sono. Isto pode ser feito por meio de diários de sono, instrumentos específicos sobre o sono ou actigrafia. A actigrafia é um método de monitoramento do sono, que é realizado por meio do actígrafo, que consiste de um monitor de atividade motora equipado com um acelerômetro, que tem a 
forma e o tamanho de um relógio e é usado habitualmente no pulso. Este método avalia indiretamente o sono por meio da quantificação e análise da atividade motora, fornecendo normalmente as seguintes medidas: 1) horário e tempo total de sono; 2) latência para o início do sono; 3) duração dos despertares noturnos; 4) número de despertares noturnos; 5) eficiência do sono (tempo efetivo de sono durante o tempo total na cama, que é calculado em porcentagem) (Pires, Vilela, \& Câmara, 2012; Souza et al., 2003).

A verificação de problemas de comportamento em crianças pode ser realizada por meio de questionários nos quais cuidadores relatam as problemáticas de suas crianças. Os questionários são amplamente utilizados tanto na pesquisa quanto na prática clínica. Estes instrumentos servem como recurso para identificação de competências e problemas comportamentais infantis, auxiliando o profissional na avaliação diagnóstica e análise funcional, no planejamento de intervenções, na avaliação de resultados e no encaminhamento (Silvares, 2000). Um dos instrumentos mais utilizados mundialmente para identificar problemas de comportamento em crianças e adolescentes por meio de informações fornecidas pelos pais é o Child Behavior Checklist (CBCL), que foi desenvolvido por Achenbach e colaboradores (Achenbach, 1991; Achenbach, 1992; Achenbach \& Edelbrock, 1983, Achenbach, Edelbrock, \& Howell, 1987; Achenbach \& Rescorla, 2000; Achenbach \& Rescorla, 2001). A bibliografia de estudos publicados utilizando o sistema Achenbach de avaliação lista mais de 8.000 publicações, relatando o uso do CBCL (Wielewicki, Gallo, \& Grossi, 2011). O instrumento se destaca das demais formas de avaliação de saúde mental pelo seu rigor metodológico, valor em pesquisas e utilidade na prática clínica. Suas escalas fornecem ampla cobertura dos sintomas psicopatológicos comumente encontrados na infância e adolescência. Os nomes atribuídos às síndromes pertencem a um vocabulário familiar aos profissionais de saúde mental para facilitar a comunicação entre aqueles que fazem uso dos inventários. O princípio de construção do $C B C L$ foi empírico, baseado no tratamento estatístico (análise fatorial) de uma lista de queixas na área de saúde mental (Duarte \& Bordin, 2000; Rocha, Araújo, \& Silvares, 2008). A versão brasileira do CBCL é denominada "Inventário de Comportamentos da Infância e Adolescência" (Emerich, Rocha, \& Silvares, 2010; Rocha, Ferrari, \& Silvares, 2011).

O CBCL foi elaborado por Achenbach, com as versões pré-escolar e escolar englobando a faixa etária de 2-3 anos e 4-18 anos respectivamente. Em 2000 a versão pré-escolar (2-3 anos) foi revisada por Achenbach e Rescorla e expandida para a versão $1 \frac{1}{2}$ a 5 anos. A atual versão pré-escolar avalia as seguintes síndromes: "reatividade emocional"; "ansiedade/depressão"; "queixas somáticas"; "problemas de atenção"; "comportamento agressivo"; e "problemas de sono”. A versão escolar 
também foi revisada por Achenbach e Rescorla em 2001 e a atual versão engloba a faixa etária de 6 a 18 anos. O instrumento avalia as síndromes: "reatividade emocional"; “ansiedade/depressão"; "queixas somáticas"; "problemas de atenção"; "comportamento agressivo"; "problemas sociais"; "problemas de pensamento" e "quebra de regras". As síndromes são avaliadas por meio de itens em que o respondente (cuidador) avalia de acordo com os seguintes critérios: não verdadeiro; um pouco verdadeiro ou algumas vezes verdadeiro; muito verdadeiro ou frequentemente verdadeiro, o que corresponde, respectivamente, a 0,1 e 2 pontos na escala.

Tanto na versão pré-escolar, como na versão escolar, a criança ou o adolescente pode ser classificado como clínico, limítrofe ou normal, tanto no que diz respeito ao funcionamento global como nos perfis internalizantes e externalizantes. As escalas referentes ao primeiro perfil são: isolamento; queixas somáticas; $e$ ansiedade/depressão. As referentes ao segundo perfil são comportamento agressivo e quebra de regras.

Compreender as possíveis repercussões que as dificuldades com o sono na infância podem acarretar nos comportamentos diurnos, pode auxiliar pesquisadores e clínicos na identificação precoce do problema, bem como no desenvolvimento de práticas preventivas e interventivas importantes. Considerando a importância do sono para o bem estar infantil, e que a má qualidade do sono pode estar associada a problemas de comportamento infantil diurno, este estudo apresenta uma revisão sobre problemas comportamentais avaliados pelo instrumento Child Behavior Checklist (CBCL) de crianças com insônia.

\section{MÉTODO}

Os estudos selecionados para revisão deste artigo foram identificados por meio das bases de dados BVS Psicologia ULAPSI Brasil, SCOPUS e PsycINFO, entre os anos 2001 e 2011, utilizando as seguintes palavras-chaves em diferentes combinações: Children, CBCL, Child Behavior Checklist, Behavior, Sleep, Sleep problems, Behavioral Insomnia, Bedtime Problems, Night Wakings.

Foram incluídos nesta revisão apenas estudos que avaliaram a relação entre problemas de sono e problemas de comportamento, avaliados pelo CBCL. Foram excluídos os estudos que não utilizaram o $C B C L$ como medida de problemas comportamentais; estudos com crianças com comprometimento neurológico ou doença crônica; estudos com crianças com problemas de sono de ordem não comportamental, como distúrbios respiratórios do sono, síndrome das pernas inquietas, narcolepsia. 


\section{RESULTADOS E DISCUSSÃO}

\section{Caracterização dos estudos}

De acordo com os critérios de inclusão e exclusão explicitados, foram selecionados 12 estudos, cujas características estão apresentadas na Tabela 1 .

Tabela 1

Características dos 12 Estudos Presentes na Revisão

\begin{tabular}{|c|c|c|c|c|c|c|}
\hline Autores & $\begin{array}{c}\text { Ano de } \\
\text { Publicação }\end{array}$ & País & $\mathbf{N}$ & Idade & Medida de sono & $C B C L$ \\
\hline Stein et al. & 2001 & EUA & 472 & $4-12$ & Questionário & $4-16$ \\
\hline Sourander & 2001 & Finlândia & 525 & 3 & Questionário & $2-3$ \\
\hline Scher et al. & 2005 & Israel & 68 & $31 / 2$ & Questionário & $2-3$ \\
\hline $\begin{array}{l}\text { Caldwell-Andrews } \\
\text { \& Kain }\end{array}$ & 2006 & EUA & 52 & 6,9 & Actigrafia & $4-18$ \\
\hline Shang et al. & 2006 & China & 1391 & $4-9$ & Questionário & $4-18$ \\
\hline Hall et al. & 2007 & Austrália & 2214 & $1-4$ & Questionário & $\begin{array}{c}2-3 \\
4-18\end{array}$ \\
\hline Gregory et al. & 2008 & Holanda & 2076 & $4-16$ & Questionário & $4-18$ \\
\hline Blunden \& Chervin & 2008 & Austrália & 80 & 9 & Questionário & $4-18$ \\
\hline Hiscock et al. & 2008 & Australia & 328 & 2 & $\begin{array}{l}\text { Relato materno: } \\
\text { Problema de sono: } \\
\text { SIM ou NÃO }\end{array}$ & $1 \frac{1 / 2}{}-5$ \\
\hline Blunden \& Chervin & 2010 & Austrália & 50 & 8,8 & Questionário & $4-18$ \\
\hline Pesonen et al. & 2010 & Finlândia & 470 & 8 & Actigrafia & $4-18$ \\
\hline Byars et al. & 2011 & EUA & 160 & $1 \frac{1 / 2-10}{2}$ & Questionário & $\begin{array}{c}1 / 2-5 \\
6-18\end{array}$ \\
\hline
\end{tabular}

Destas 12 publicações, quatro eram da Austrália, três dos EUA, duas da Finlândia, uma da China, uma da Holanda e uma de Israel. As publicações foram realizadas entre os anos de 2001 e 2011, sendo que três estudos foram publicados entre 2001 e 2005, metade dos estudos entre 2006 e 2009 e três entre 2010 e 2011.

Os estudos envolveram amostras de tamanhos muito variados, de modo que a maior amostra é 44 vezes superior à menor. A idade dos participantes variou de 1 a 16 anos, predominando crianças em idade pré-escolar e escolar.

$\mathrm{Na}$ presente revisão, os estudos utilizaram diferentes medidas para avaliar o sono das crianças. 


\section{Medidas de Avaliação do Sono e Critérios para Insônia}

No que se refere às medidas de avaliação de sono, nove estudos utilizaram questionários e inventários específicos sobre o sono da criança, dois utilizaram a actigrafia e um o relato materno identificando se a criança tinha problemas de sono (sim ou não). A descrição dos critérios para insônia, bem como as medidas de avaliação de sono estão descritas a seguir.

A medida de sono utilizada no estudo de Stein, Mendelsohn, Obermeyer, Amromin e Benca (2001). foi o Sleep Behavior Questionnaire. Trata-se de um questionário, desenvolvido pelos autores e que é composto de 25 itens que exploram hábitos de sono (sestas diurnas; hora de dormir e acordar) e problemas de sono, tais como dificuldade de iniciar o sono; frequentes despertares noturnos; enurese; ressonar e falar dormindo. Tais características são avaliadas de acordo com sua frequência (nunca; menos do que uma vez na semana; pelo menos uma vez na semana; toda noite e não sei) considerando os últimos seis meses. A insônia foi definida pelos autores como dificuldade de adormecer (com latência superior a 30 minutos) e frequentes despertares durante a noite, em pelo menos duas semanas nos últimos seis meses.

Três estudos utilizaram o CBCL como medida para avaliar o sono das crianças. O estudo de Gregory, Ende, Willis e Verhulst, (2008) utilizou a versão 4-18 anos do $C B C L$ na qual as questões relacionadas ao sono referem-se a: dormir menos que outras crianças; cansaço durante o dia; dificuldade para dormir; pesadelos; dorme mais do que a maioria das crianças e fala enquanto dorme. A insônia, em particular, compreende os itens dormir menos do que outras crianças; cansaço durante o dia e dificuldade para dormir. Sobre esses itens, os pais foram instruídos a responder em uma escala em que 0 correspondia a ausência do problema, 1 a problema ocasional ou 2 a problema frequente. Os itens foram considerados problemas quando os pais respondiam problema ocasional ou problema frequente.

Sourander (2001) e Hall, Zubrick, Silburn, Parsons e Kurinczuk, (2007) utilizaram a versão pré-escolar (2-3 anos) do $C B C L$, que contém questões como não dormir sozinho; resiste ir para cama no momento de dormir e frequentes despertares noturnos. Para definição de problema de sono, o estudo de Sourander (2001), considerou resistência em ir para cama; despertar noturno e não dormir sozinho e as questões se referiam à ocorrência destes problemas no momento atual ou nos últimos dois meses. O estudo de Hall et al. (2007) apontou que altas pontuações nestes itens se associavam a problemas de sono, no entanto os autores não definiram um ponto de corte que definisse crianças com ou sem problemas de sono. Somado aos itens do CBCL, Hall et al. (2007) também utilizaram a dimensão Sleep Rhythmicity da escala Dimensions of Temperament Survey (DOTS-R) Child Version, para avaliar o sono das crianças. Esta dimensão é composta de seis itens, tais como "meu filho 
usualmente tem a mesma quantidade de sono toda noite"; "meu filho vai dormir no mesmo horário todas as noites".

Scher, Zukerman e Epstein (2005) utilizaram o Sleep Questionnaire para avaliação da insônia. Este questionário, semiestruturado, desenvolvido por estes autores, é composto por itens que descrevem a rotina de sono da criança; hábitos no momento de dormir; frequência de "despertares noturnos"; tempo que a criança leva para voltar a adormecer após o despertar durante a noite e duração do sono. A primeira dimensão do instrumento refere-se à resposta da mãe quanto à presença de problemas de sono da criança, que era avaliada de acordo com a seguinte pontuação: 0 = não tem problema, 1 = tem um problema (dificuldade de adormecer ou despertar noturno), 2 = tem ambos os problemas (dificuldade de adormecer $e$ despertar noturno). A segunda dimensão tinha como objetivo caracterizar dois aspectos do sono: um relativo ao seu padrão (com questões referentes ao momento de ir para cama; tempo para adormecer) e outro relativo aos despertares (número de noites na semana com sono interrompido; número de despertares; duração dos despertares). A gravidade desses aspectos era avaliada de acordo com o critério de Richman (1981) com a utilização de uma escala em que a pontuação 0 referia-se a ausência do problema e 4 a problema muito severo. Para avaliar o padrão de sono foram incluídos 3 itens: tempo para ir para cama; latência de início de sono e duração total de sono. $\mathrm{O}$ aspecto do sono relativo aos despertares incluía número de interrupções de sono durante a semana; número de despertares durante a noite e tempo de cada despertar. Com base na média dos dois aspectos (padrão de sono e despertares) foi utilizada uma pontuação composta que definiu crianças com e sem problemas de sono. Pontuações acima de 1.50 (em uma escala de 0-4) representavam crianças com problemas de sono.

O instrumento Sleep Habits and Problems foi utilizado no estudo de Shang et al. (2006). Trata-se de um questionário respondido pelos pais sobre hábitos e problemas de sono de seus filhos presentes no último mês. A insônia comportamental foi definida como a) Insônia inicial, compreendida como latência de início de sono superior a meia hora, em pelo menos 3 noites na semana; b) Dormir tarde, que compreende dormir após a meia noite pelo menos uma vez na semana; c) Despertar noturno, que compreende o despertar e ficar acordado pelo menos meia hora em uma frequência de 3 vezes na semana.

O inventário The Sleep Disturbance Scale for Children (SDSC), utilizado nos estudos de Blunden e Chervin $(2008,2010)$, é um instrumento com questões fechadas, de autopreenchimento, elaborado por Bruni et al. (1996). É composto por itens referentes aos comportamentos relacionados ao sono de crianças e mede diferentes problemas de sono, por meio de subescalas (dificuldades de iniciar e manter o sono; distúrbios respiratórios do sono; distúrbios do despertar; distúrbios da transição 
sono-vigília; sonolência excessiva; hiperhidrose). Cada item é anotado em uma escala que varia de 1 a 5 ( 1 =nunca; 5 = sempre $)$. A classificação para problemas de sono, tanto para soma total quanto para cada subescala, ocorre quando a pontuação T é igual ou superior a 60. Respostas que referem ocorrência nos itens a criança não quer ir para cama; a criança tem dificuldade para adormecer; a criança está agitada antes de dormir; a criança desperta mais de 2 vezes durante a noite; a criança tem dificuldade de adormecer após acordar durante a noite; sonolência diurna são consideradas para subescala "dificuldade de iniciar e manter o sono". Para subescala "distúrbios respiratórios do sono", são consideradas as respostas que referem ocorrência nos itens dificuldades respiratórias; apneia do sono e ressonar. A subescala "distúrbios do despertar" abrange a ocorrência de respostas nos itens sonambulismo; pesadelos e terror noturno. Respostas que referem ocorrência nos itens: distúrbios rítmicos do movimento; alucinações hipnagógicas; movimentação noturna; falar dormindo e bruxismo, são consideradas para subescala "distúrbios da transição sono-vigília". A subescala "sonolência excessiva diurna" abrange ocorrência nas questões dificuldade de acordar; acordar cansado; paralisia do sono; sonolência diurna. Já a subescala "hiperhidrose do sono" abrange a ocorrência nos itens adormecer suado e transpirar durante a noite.

O estudo de Byars, Yeomans-Maldonado e Noll (2011) utilizou o instrumento The Children's Sleep Habits Questionnaire, que é composto de 33 itens que avalia os hábitos e distúrbios de sono da criança, a partir de oito subescalas ("resistência para dormir"; "despertar noturno"; "duração do sono"; "latência de início do sono"; "cansaço diurno"; "parassonias"; “distúrbios respiratórios do sono" e "ansiedade relacionado ao sono"). Os itens das subescalas devem ser respondidos pelos cuidadores a partir de uma escala Likert de 1 a 3 ( $1=0$ a 1 vez por semana; $2=2$ a 4 vezes por semana; 3 = frequentemente, 5 a 7 vezes por semana) de acordo com sua frequência. Pontuações mais altas representam alta frequência de dificuldades com o sono sendo que uma pontuação $T$ igual ou maior que 41 representa que a criança apresenta problemas de sono. A insônia é identificada por meio de itens que exploram problemas como resistência para dormir; despertar noturno; duração do sono; latência para início do sono; cansaço diurno e ansiedade noturna.

O critério para problema de sono no estudo de Hiscock, Bayer, Hampton, Ukoumunne e Wake (2008) foi o relato materno identificando a presença (sim ou não) de contínuos problemas de sono (despertar noturno e dificuldade de adormecer) nas duas últimas semanas.

A actigrafia, foi utilizada nos estudos de Caldwell-Andrews e Kain (2006) e Pesonen et al. (2010). Problemas de sono identificados pelo actígrafo levam em consideração: a duração do sono; eficiência do sono diminuída; número de despertares; latência de início do sono. O estudo de Caldwell-Andrews e Kain (2006) utilizou o 
actígrafo ACTME (Basic Motionlogger Actigraph Ambulatory Monitoring Inc., Ardsley, NY, EUA). Os dados do actígrafo foram coletados durante 16 dias em combinação com diários de sono. Os critérios para problemas de sono consideraram a baixa eficiência de sono (percentagem baixa do tempo total de sono real durante o período total na cama) das crianças. Pesonen et al. (2010) utilizou o actígrafo Actiwatch AW4 (Cambridge Neurotechnology Ltd., Reino Unido). Os dados do actígrafo foram coletados durante 7 dias. O estudo considerou como principal critério para problema de sono a curta duração de sono, que é definida como quantidade média de sono inferior a 7,7 horas. O critério para curta duração de sono, teve como base os estudos de Nixon et al. (2008) e Owens, Mehlenbeck, Lee e King (2008).

Entre as diferentes medidas para avaliar o sono da criança, predominou a utilização de questionários/inventários. A escolha da medida de avaliação é de extrema importância, uma vez que a partir de suas propriedades é que são definidos os critérios de inclusão para o estudo. A presente revisão demonstrou que apesar da variedade de instrumentos utilizados para avaliar o sono infantil, a maioria das medidas utilizadas identificavam os mesmos padrões de sono (dificuldade de iniciar e manter o sono; curta duração de sono; sonolência durante o dia; adormecer apenas na presença parental; tempo para adormecer) em frequência (ocorrência durante a semana) similar. Todavia, alguns estudos consideraram a presença dos sintomas no momento atual, enquanto outros estudos consideraram que os problemas de sono deveriam perdurar por um determinado tempo (seis meses). Desta forma, não ficou claro durante quanto tempo (em meses) estes sintomas devem ocorrer para ser critério para insônia.

\section{Child Behavior Checklist (CBCL)}

Diferentes versões do $C B C L$ foram utilizadas nos estudos analisados. Por meio da Tabela 1 , podemos verificar que a maioria $(N=7)$ dos estudos utilizou o $C B C L$ (4-18). A diversidade de versões do $C B C L$ existe devido a constante revisão do instrumento e sua adaptação conforme a faixa etária. O desenvolvimento do $C B C L$ iniciou-se em meados da década de 60, com o interesse do pesquisador Achenbach em investigar a diversidade de sintomas/queixas que poderiam levar crianças para tratamento psiquiátrico. $\mathrm{O}$ desenvolvimento desse instrumento teve inicio com a produção de uma lista preliminar de comportamentos de crianças coletados em registros de casos clínicos, posteriormente adaptada para recolher informações dos pais (Bordin et al., no prelo).

O perfil de comportamento da criança quando avaliada pelo CBCL foi inicialmente padronizado para meninos de 6 a 11-anos, e mais tarde, expandido para ambos os sexos na faixa etária 6-16 anos. Em 1983, Achenbach e Edelbrock publicaram 
o "Manual de Comportamento Infantil Checklist/4-16", para avaliar crianças em idade escolar, de 4 a 16 anos. Em 1991 uma versão revista do CBCL foi publicada para 4-18 anos sem alterações significativas no teor de itens em relação à versão de 1983. Nesta versão, as mudanças mais relevantes que envolvem o perfil do comportamento da criança em idade escolar incluíram o estabelecimento de uma faixa limítrofe para escalas de pontuações $\mathrm{T}$ e o desenvolvimento de formatos para pais e professores (Bordin et al., no prelo). Em 2001, Achenbach e Rescorla revisaram a versão escolar, para ser aplicada na faixa etária de 6-18 anos. Foram substituídos e adicionados alguns itens. Foram adicionados os itens sobre consumo de álcool e tabaco e ruptura de regras. A síndrome "comportamento delinquente" foi substituída por "quebra de regras". Foram reformulados itens para problemas de atenção, e a síndrome "retraimento" foi substituída pela síndrome "retraimento/depressão".

A versão de crianças de dois e três anos (1987, 1992), foi elaborada por Achenbach, e parte (59 itens) de sua construção foi baseada na versão CBCL (4-18 anos) e os demais itens foram desenvolvidos especificamente para faixa etária de 2 e 3 anos. Em 2000, Achenbach e Rescorla revisaram a versão pré-escolar, ampliando sua faixa etária para 11/2-5 anos.

Os dados do CBCL obtidos nas 12 pesquisas desta revisão, foram analisados e os resultados demonstraram associações significativas entre insônia e problemas de comportamento. As relações entre insônia e problemas comportamentais foram organizadas por tópicos (Insônia e Problemas Externalizantes, Insônia e Problemas Internalizantes e Insônia e Outros Problemas de Comportamento) que são descritos a seguir.

\section{Sintomas de Insônia e Problemas Externalizantes}

Grande parte $(N=10)$ das publicações analisadas pelo presente estudo encontraram associações entre sintomas de insônia e problemas externalizantes. O comportamento de agressividade e de quebrar regras ou comportamento delinquente esteve consistentemente presente nas crianças com problemas de sono na presente revisão. Um exemplo disto é o estudo de Byars et al. (2011) que observou crianças com problemas de sono avaliados pelo Children's Sleep Habits Questionnaire na faixa etária entre $1 \frac{1}{2}$ e 10 anos e constatou que $34 \%(N=53)$ destas apresentavam problemas externalizantes de comportamento.

Correlações significativas entre sintomas de insônia e comportamento agressivo (0.38; $\mathrm{p}<0.001)$, comportamento delinquente $(0.22 ; p<0.001)$ e total de problemas externalizantes $(0.40 ; p<0.001)$ foram encontradas por Stein et al. (2001) ao investigar a prevalência de problemas de sono em 412 crianças por meio do Sleep Behavior Questionnaire e suas associações com sintomas psicopatológicos avaliados pelo $C B C L$. 
Com o objetivo de investigar sono e problemas comportamentais na infância, por meio do questionário Sleep Habits and Problems, Shang, Gau e Soong, (2006) apontaram que crianças que dormiam tarde, que despertavam bastante durante a noite e que tinham dificuldade em adormecer pontuaram mais nas escalas de comportamento delinquente e comportamento agressivo $(p<0.05)$ do que as crianças do grupo controle. A análise de regressão demonstrou ainda que o comportamento agressivo foi um dos comportamentos mais frequentes associados com problemas de sono referentes a despertares noturnos e dormir tarde $(p<0.0001)$.

Na pesquisa de Blunden e Chervin (2008) com crianças que frequentam programas psicoeducacionais, as análises mostraram correlações significativas entre problemas com iniciar ou manter o sono e sonolência diurna avaliados pelo Sleep Disturbance Scale for Children e comportamento externalizante $(p<0.05)$. A análise de regressão, controlada por sexo, idade e escolaridade da mãe mostrou um aumento de probabilidade (cinco vezes mais) de distúrbios de sono em crianças classificadas como limítrofes e clínicas no CBCL. Em estudo realizado pelos mesmos autores em 2010, o item acordar frequentemente durante a noite avaliado pelo Sleep Disturbance Scale for Children, foi correlacionado com os índices do CBCL: total de problemas de comportamento externalizantes $(r=0.32 ; p=0.02)$ e comportamento de agressão $(r=0.37 ; p=0.009)$ em 50 crianças indígenas e não indígenas com idade média de 8,8 anos.

Dormir com os pais também foi associado com problemas externalizantes, como demonstrado no estudo de Sourander (2001) com 525 crianças com 3 anos de idade. O estudo avaliou problemas de comportamento e de sono por meio do CBCL, e seus resultados demonstraram que os problemas de sono estavam associados com dormir com os pais $(p<0.001)$. Além disso, partilhar o quarto com os pais foi associado com escores altos no perfil externalizante (14.4; $p<0.05)$.

Associações longitudinais entre sono e problemas externalizantes também foram encontradas. O estudo de Hall et al. (2007), com 2214 crianças avaliou o sono de crianças nos 3 primeiros anos de vida por meio do Sleep Rhythmicity e depois examinou as relações entre problemas de sono aos 3 anos e comportamento agressivo aos quatro anos, por meio do $C B C L$. Os resultados revelaram que escores mais altos de problemas de sono aos 3 anos são preditores de comportamento agressivo aos 4 anos $(t=9.15 ; p<0.001$, effect size $=0.05)$.

Gregory et al. (2008) também encontraram em seu estudo problemas de sono como fator de risco para o comportamento agressivo. A pesquisa foi composta por uma amostra de 2076 crianças inicialmente com idades de 4 a 16 anos. A avaliação de sono foi realizada em 5 momentos durante o desenvolvimento das crianças, por meio do CBCL. Posteriormente foram avaliados os comportamentos dos participantes em idade adulta (18 a 32 anos) por meio do Young Adult Self-Report (YSR). A análise 
dos dados revelou que dormir pouco na infância constitui fator de risco para comportamentos de agressividade (1.51; 95\% IC, 1.13 - 2.02; $P=0.05)$ na idade adulta.

Poucos estudos avaliaram o sono por meio de medida objetiva, como a actigrafia. Um desses estudos é a pesquisa de Caldwell-Andrews et al. (2006) que avaliaram os preditores psicológicos de problemas de sono em 52 crianças com idade média de 6,9 anos no pós-operatório ambulatorial. O sono foi avaliado por meio de actígrafo e o comportamento agressivo por meio do CBCL. Os resultados demonstraram que crianças com pontuações mais elevadas de comportamento agressivo foram significativamente mais propensas a experimentar menor eficiência de sono após a cirurgia $(88.9 \pm 5,2 \%$ vs $77.4 \pm 11,5 \% ; P=0.021)$. O comportamento agressivo da criança ( $C B C L$ externalização) constituiu preditor significativo de má qualidade de sono no pós-operatório.

Pesonen et al. (2010) também utilizaram a actigrafia para avaliar o sono. O estudo explorou associações entre duração de sono e problemas emocionais e comportamentais em crianças de 8 anos. Os dados mostraram que crianças que dormiam pouco aos fins de semana apresentaram riscos 2,9 vezes maiores de presença de problemas externalizantes $(p=0.05)$. A curta duração de sono aos fins de semana também foi associada com maiores chances de comportamento de quebrar regras $(\mathrm{OR}=3.9$, IC 95\% $1.1-12.4=0.04)$.

\section{Sintomas de Insônia e Problemas Internalizantes}

Metade das publicações $(N=6)$ apresentou em seus resultados relações entre problemas de sono e problemas internalizantes na população infantil. Por exemplo, o estudo de Byars et al. (2011), com uma amostra composta por 156 crianças com sintomas de insônia , 40\% $(N=63)$ eram classificadas como "clínicas" para problemas internalizantes.

No estudo de Stein et al. (2001), com crianças na faixa etária de 4 a 12 anos, sintomas de insônia foram altamente correlacionados com pontuações nas síndromes de "isolamento" $(0.27 ; p<0.001)$, “queixas somáticas" $(0.26 ; p<0.001)$, “ansiedade/depressão" $(0.39 ; p<0.001)$ e "total de problemas internalizantes" $(0.40$ $p<0.001)$. Além disso, a análise de regressão revelou que pontuações nas síndromes de "ansiedade/depressão" ( $\left.F=65.9 ; R^{2}=0.15 ; p<0.001\right)$ e "queixas somáticas" ( $F$ $\left.=30.2 ; R^{2}=0.19 ; p<0.001\right)$ prediziam problemas relacionados à insônia. Assim, crianças com queixas somáticas e sintomas de ansiedade e depressão tiveram mais dificuldade para iniciar e manter o sono.

Em pesquisa realizada com 1391 crianças de 4 a 9 anos, Shang et al. (2006) demonstraram que, comparadas a outras crianças, aquelas com sintomas de insônia inicial, 
que dormiam tarde e que despertavam frequentemente a noite tiveram pontuações significativamente $(p<0.05)$ mais altas nas síndromes "ansiedade/depressão", "queixas somáticas" e "isolamento". Resultados obtidos por meio de análise de regressão mostraram que sintomas de ansiedade/depressão e isolamento prediziam despertares noturnos ( $p<0.05 ; p<0.01$ respectivamente) e queixas somáticas prediziam sintomas de insônia inicial $(p<0.05)$ e dormir tarde $(p<0.0001)$.

Blunden e Chervin (2008) encontraram correlações significativas $(p<0.0001)$ entre problemas internalizantes e problemas de inicio e manutenção do sono, despertares noturnos e sonolência diurna em 80 crianças com idade média de 9 anos. Problemas com o sono também foram identificados como fator de risco para problemas internalizantes $(p<0.05)$. Em outra pesquisa realizada por Blunden e Chervin (2010), com crianças australianas indígenas e não indígenas, os problemas de despertares noturnos foram positivamente correlacionados com escores na síndrome "isolamento" $(r=0.31 ; p=0.02)$ para as duas populações. Os autores também encontraram correlações entre sonolência diurna e isolamento $(r=0.39$; $p<0.05)$ para as crianças indígenas.

Além de destacar as associações simultâneas entre sintomas de insônia e problemas de comportamento, pesquisadores vêm investigando também associações longitudinais. Um exemplo disto é o estudo de Gregory et al. (2008) no qual foram investigadas associações entre problemas de sono durante a infância, identificados por questões do $C B C L$, e subseqüentes dificuldades emocionais e comportamentais, avaliadas por meio do instrumento YSR. Os resultados demonstraram que crianças e adolescentes que dormiam menos horas durante a fase de desenvolvimento, na idade adulta apresentaram maior probabilidade de pontuar nas escalas "ansiedade/ depressão" (OR = 1.43; IC 95\% 1.07-1.90; $P=.01)$.

\section{Sintomas de Insônia e Outros Problemas de Comportamento}

Além dos comportamentos internalizantes (queixas somáticas, ansiedade/depressão, retraimento) e externalizantes (agressividade e quebra de regras), outros problemas de comportamento foram associados a sintomas de insônia no presente trabalho. Tais problemas referem-se às seguintes síndromes do CBCL: "problemas de atenção", "problemas de pensamento" e "problemas sociais". Associações entre problemas de atenção e sono foram encontradas em quatro estudos, os problemas de pensamento e sono em três e a relação entre problemas sociais e sono foi encontrada em dois estudos.

Pesquisadores (Stein et al., 2001) encontraram associações significativas ( $p<$ 0.001) entre problemas de sono (sintomas de insônia e sonolência diurna) e problemas de atenção, problemas de pensamento e problemas sociais. Uma análise de 
regressão realizada nesta pesquisa indicou que problemas de atenção podem predizer problemas de iniciar e manter o sono $\left(F=43.0 ; R^{2}=0.18 ; p<0.001\right)$ e que os problemas sociais podem predizer cansaço diurno $\left(F=48.9 ; R^{2}=0.11 ; p<0.001\right)$. Em outro estudo (Shang et al., 2006), comparadas a crianças controle, crianças que dormiam tarde, que despertavam com frequência a noite e que tinham insônia inicial tiveram pontuações significativamente $(p<0.05)$ mais altas nas síndromes "problemas de atenção", "problemas de pensamento" e "problemas sociais". Além disso, uma análise de regressão apontou que "problemas de pensamento" $(p<0.01)$ constituíam fator de risco para sintomas de insônia inicial.

Dificuldade de atenção, presente na síndrome "problemas de atenção" do $C B C L$, foi relatado no estudo de Blunden e Chervin (2008), que demonstrou correlações significativas entre problemas de atenção e problemas comportamentais de sono $(0.65 ; p<0.0001)$, despertar noturno $(0.64 ; p<0.0001)$ e sonolência diurna $(0.46 ; p$ $<0.0001)$. Ainda neste contexto, os resultados da pesquisa de Pesonen et al. (2010) apontaram que crianças que dormiam pouco apresentaram maior probabilidade para problemas de atenção (OR 3.3; 95\% IC 1.2 a $8.8 ; p=0.02$ ) e problemas de pensamento (OR 4.0; 95\% IC 1.6 a10.1; $p=0.003$ ).

A má qualidade de sono como preditora de problemas de comportamento foi demonstrada no estudo de Scher, Zukerman e Epstein (2005) que teve como objetivo avaliar se persistentes dificuldades com o sono durante a infância poderiam constituir um precursor de problemas comportamentais posteriores. O sono das crianças foi avaliado pelo Sleep Questionnaire aos 3, 6, 9 e 12 meses e depois novamente aos 42 meses, e o CBCL foi aplicado nas 68 mães quando a criança tinha 42 meses. Os resultados revelaram que crianças com pobre qualidade de sono (frequente despertar noturno, curta duração de sono, dificuldade de adormecer) obtiveram pontuações mais altas no $C B C L(t=2.35 ; p<0.05)$ quando comparadas com crianças que dormiam bem.

A maioria $(N=11)$ das pesquisas desta revisão encontrou associações entre problemas de sono e problemas de comportamento, fossem eles externalizantes, internalizantes ou outros problemas de comportamento avaliados pelo $C B C L$, com exceção de uma pesquisa, efetuada por Hiscock et al. (2008) que teve como objetivo avaliar os problemas comportamentais das crianças aos 2 anos de idade após serem submetidas a uma intervenção comportamental para problemas de sono infantil quando eram bebês. Aos 7 meses, o sono das crianças era avaliado por meio de relato materno, dos 8 aos 10 meses as mães recebiam orientações para o manejo de problemas com o sono na infância e aos 24 meses o comportamento das crianças era avaliado pelo CBCL. Os resultados demonstraram que não houve associação entre problemas de sono e problemas de comportamento, pois tanto as crianças que tiveram melhoras no sono, como as que não tiveram, não apresentaram problemas de comportamento, avaliados pelo CBCL. 


\section{CONSIDERAÇÕES FINAIS}

A presente revisão da literatura demonstra que o aumento de interesse nas relações entre problemas de sono e de comportamento se justifica. De fato, os problemas do sono têm sido associados com uma série de outras dificuldades comportamentais e emocionais concorrentemente e também podem representar indicadores de risco para o surgimento de problemas comportamentais e emocionais na vida adulta. Essas associações são demonstradas quando avaliadas simultaneamente e também em estudos ao longo do tempo. Associação entre problema de sono e de comportamento é provavelmente bidirecional, de modo que os problemas de sono, ou o sono insuficiente pode exacerbar problemas de comportamento como também os problemas de comportamento podem prejudicar os padrões de sono.

O CBCL como medida para avaliar o comportamento mostra-se satisfatório, uma vez que é possível coletar diferentes domínios de problemas comportamentais, como problemas internalizantes, externalizantes e demais síndromes. Contudo, a diversidade de definições dos problemas de sono pode representar uma dificuldade. Para um melhor detalhamento dos padrões de sono das crianças, pesquisas posteriores poderiam combinar a utilização de medidas subjetivas (questionários, inventários, diários) e objetivas, como o actígrafo.

Além disso, pesquisas adicionais são necessárias para explorar os outros fatores que podem estar associados aos problemas de sono e de comportamento. Entre esses fatores encontram-se a influência da faixa etária nos problemas de sono e de outros problemas de comportamento e os fatores ambientais como a relação parental e a cultura familiar e da comunidade onde a criança está inserida.

Espera-se que este estudo possa estimular a produção de pesquisas sobre o tema, servindo como incentivo para o desenvolvimento de práticas preventivas e interventivas para os problemas de sono em crianças.

\section{REFERÊNCIAS}

Achenbach, T. M. (1991). Integrative Guide for the 1991 CBCL 4/18, YSR, and TRF Profiles. Burlington, V.T.: University of Vermont, Department of Psychiatry.

Achenbach, T. M. (1992). Manual for the Child Behavior Checklist/2-3 and 1992 Profile. Burlington, V.T.: University of Vermont, Department of Psychiatry.

Achenbach, T. M., \& Edelbrock T. (1983). Manual for the Child Behavior Check List and Revised Child Behavior Profile. Burlington, VT: University of Vermont.

Achenbach, T. M., Edelbrock, C., \& Howell, C. T. (1987). Empirically based assessment of the behavioral/ emotional problems of 2-and 3-year-old children. Journal of Abnormal Child Psychology, 15, 629-650. 
Achenbach, T. M., \& Rescorla, L. A. (2000). Manual for ASEBA Preschool Forms \& Profiles. Burlington, V.T.: University of Vermont, Research Center for Children, Youth, \& Families.

Achenbach, T. M., \& Rescorla, L. A. (2001). Manual for ASEBA School-Age Forms \& Profiles. Burlington, V.T.: University of Vermont, Research Center for Children, Youth, \& Families.

American Academy of Sleep Medicine. (2005). International Classification of Sleep Disorders: Diagnostic and Coding Manual, $2^{\text {nd }}$ ed. Westchester, IL: American Academy of Sleep Medicine.

Blunden, S. L., \& Chervin, R. D. (2008). Sleep problems are associated with poor outcomes in remedial teaching programmes: a preliminary study. Journal of Paediatrics and Child Health, 44, 237-242.

Blunden, S. L., \& Chervin, R. D. (2010). Sleep, performance and behavior in Australian indigenous and non-indigenous children: an exploratory comparison. Journal of Paediatrics and Child Health, 46, 10-16.

Bordin, I. A., Rocha, M. M., Paula, C. S., Teixeira, M. C. T. V., Achenbach, T. M., Rescorla, L. A., \& Silvares, E. F. M. (no prelo). Child Behavior Checklist/CBCL, Youth Self-Report/YSR and Teacher's Report Form/TRF: an overview of the development of original and Brazilian versions. Cadernos de Saúde Pública.

Bruni, O., Ottaviano, S., Guidetti, V., Romoli, M., Innocenzi, M., Cortesi, F., \& Giannotti F. (1996). The Sleep Disturbance Scale for Children (SDSC) construction and validation of an instrument to evaluate sleep disturbances in childhood and adolescence. Journal of Sleep Research, 5, 251-261.

Byars, K. C., Yeomans-Maldonado, G., \& Noll, J. G. (2011). Parental functioning and pediatric sleep disturbance: an examination of factors associated with parenting stress in children clinically referred for evaluation of insomnia. Sleep Medicine, 12, 898-905.

Caldwell-Andrews, A. A., \& Kain, Z. N. (2006). Psychological predictors of postoperative sleep in children undergoing outpatient surgery. Pediatric Anesthesia, 16, 144-151.

Duarte, C. S., \& Bordin, I. A. S. (2000). Instrumentos de Avaliação. Revista Brasileira de Psiquiatria, 22, 55-58.

Emerich, D. R., Rocha, M. M., \& Silvares, E. F. M. (2010). Estudo de validação preliminar do "Inventário dos Comportamentos de Crianças e Adolescentes de 6 a 18 anos" (CBCL/6-18), Versão Brasileira do Child Behavior Checklist. Projeto de Pesquisa em Andamento no Instituto de Psicologia da Universidade de São Paulo sob Coordenação de E.F.M. Silvares.

Gregory, A. M., Ende, J. V., Willis, T. A., \& Verhulst, F. C. (2008). Parent-reported sleep problems during development and self-reported anxiety/depression, attention problems, and aggressive behavior later in life. Archives of Pediatrics and Adolescent Medicine, 162(4), 330-335.

Gregory, A. M., \& Sadeh, A. (2012). Sleep, emotional and behavioral difficulties in children and adolescents. Sleep Medicine Reviews, 16, 129-136.

Hall, W. A., Zubrick, S. R., Silburn, S. R., Parsons, D. E., \& Kurinczuk, J. J. (2007). A model for predicting behavioural sleep problems in a random sample of Australian preschoolers. Infant and Child Development, 16, 509-523.

Hill, C. (2011). Practitioner review: effective treatment of behavioural insomnia in children. Journal of Child Psychology and Psychiatry, 52(7), 731-741.

Hiscock, H., Bayer, J. K., Hampton, A., Ukoumunne, O. C., \& Wake, M. (2008). Long-term mother and child mental health effects of a population - based infant sleep intervention: cluster-randomized, controlled trial. Pediatrics, 122, 621-627.

Meltzer, L. J., \& Mindell, J. A. (2008). Behavioral sleep disorders in children and adolescents. Sleep Medicine Clinics, 3, 269-279.

Mindell, J., \& Owens, J. (2010). A clinical guide to pediatric sleep. Philadelphia: Lippincott, Williams, and Wilkins. 
Nixon, G. M., Thompson, J. M., Han, D. Y., Becroft, D. M., Clark, P. M., Robinson, E., Waldie, K. E., Wild, C. J., Black, P. N., \& Mitchell, E. A. (2008). Short sleep duration in middle childhood: Risk factors and consequences. Sleep. 31, 71-78.

Owens, J. A., Mehlenbeck, R., Lee, J., \& King, M. M. (2008). Effect of weight, sleep duration, and comorbid sleep disorders on behavioral outcomes in children with sleep-disordered breathing. Archives of Pediatrics and Adolescent Medicine, 162, 313-321.

Owens, J. A., \& Mindell, J. A. (2011). Pediatric insomnia. Pediatric Clinics of North America, 58, 555-569.

Pesonen, A. K., Raikkonen, K., Paavonen, E. J., Heinonen, K., Komsi, N., Lahti, J., Kajantie, E., Jarvenpaa, A. L., \& Strandberg, T. (2010). Sleep duration and Regularity are associated with behavioral problems in 8 year-old children. International Journal of Behavioral Medicine, 17, 298-305.

Pires, M. L. N., Vilela, C. B., \& Câmara, R. L. (2012). Desenvolvimento de uma medida de hábitos de sono e aspectos da prevalência de problemas comportamentais de sono na infância: uma contribuição. In N. Silva-Filho, D. P. A. Ribeiro, \& H. R. Rosa (Eds.), Processos Clínicos e Saúde Mental (pp. 190-196). São Paulo: Vetor Editora Psico-Pedagógica.

Richman, N. (1981). A community survey of characteristics of one- to two-year-olds with sleep disruptions. Journal of the American Academy of Child Psychiatry, 20, 281-291.

Rocha, M. M., Araújo, L. G. S., \& Silvares, E. F. M. (2008). Um estudo comparativo entre duas traduções brasileiras do Inventário de Autoavaliação para Jovens (YSR). Psicologia: Teoria e Prática, 10(1), 14-24.

Rocha, M. M., Ferrari, R. A., \& Silvares, E. F. M. (2011). Padrões de concordância entre múltiplos informantes na avaliação dos problemas comportamentais de adolescentes: implicações clínicas. Estudos e Pesquisas em Psicologia (Online), 11, 948-964.

Scher, A., Zukerman, S., \& Epstein, R. (2005). Persistent night waking and settling difficulties across the first year: early precursors of later behavioural problems? Journal of Reproductive and Infant Psychology, 23(1), 77-88.

Shang, C. H., Gau, S. S. F., \& Soong, W. T. (2006). Association between childhood sleep problems and perinatal factors, parental mental distress and behavioral problems. Journal of Sleep Research, 15, 63-73.

Silvares, E. F. M. (2000). Avaliação e intervenção clínica comportamental infantil. In E. F. M. Silvares. (Ed.), Estudos de caso em Psicologia Clínica Comportamental. (pp. 13-30), 1ª ed., v.1. São Paulo: Papirus.

Sourander, A. (2001). Emotional and behavioural problems in a sample of finish three-year-olds. European Child \& Adolescent Psychiatry, 10, 98-104.

Souza, L., Benedito-Silva, A. A., Pires, M. L. N., Poyares, D., Tufik, S., \& Calil, H. M. (2003). Further validation of actigraphy for sleep studies. Sleep, 26, 81-85.

Stein, M. A., Mendelsohn, J., Obermeyer, W. H., Amromin, J., \& Benca, R. (2001). Sleep and behavior problems in school-aged children. Pediatrics, 107(4), 60.

Wielewicki, A., Gallo, A. E., \& Grossi, R. (2011). Instrumentos na prática clínica: CBCL como facilitador da análise funcional e do planejamento da intervenção. Temas em Psicologia, 19(2), 513-523.

Witcher, L. A., Gozal, D., Molfese, D. M., Salathe, S. M., Spruyt, K., \& Crabtree, V. M. (2012). Sleep hygiene and problem behaviors in snoring and non-snoring school-age children. Sleep Medicine, 13, 802-809. 